\title{
Constraining top quark flavor violation and dipole moments through three and four top quark productions at the LHC
}

\author{
Malihe Malekhosseini, ${ }^{1, *}$ Mehrdad Ghominejad, ${ }^{1, \dagger}$ Hamzeh Khanpour, ${ }^{2,3, \$}$ and Mojtaba Mohammadi Najafabadi ${ }^{3, \S}$ \\ ${ }^{1}$ Faculty of Physics, Semnan University, Semnan P.O.Box 35131-19111, Semnan, Iran \\ ${ }^{2}$ Department of Physics, University of Science and Technology of Mazandaran, \\ P.O.Box 48518-78195, Behshahr, Iran \\ ${ }^{3}$ School of Particles and Accelerators, Institute for Research in Fundamental Sciences (IPM), \\ P.O.Box 19395-5531, Tehran, Iran
}

(Received 15 July 2018; published 1 November 2018)

\begin{abstract}
In this paper, we examine the sensitivity of the three top quark production at the LHC to the top quark flavor-changing neutral currents (FCNC) as well as the sensitivity of the four top production to the strong and weak dipole moments of the top quark. Upper limits at 95\% CL on the branching fractions of $\mathcal{B}(t \rightarrow q X)$, where $X=g, Z, \gamma, H$ and $q=u, c$, are set by performing an analysis on three top events in the same-sign dilepton channel. We consider the main sources of the background processes and a realistic detector simulation is performed at the center-of-mass energy of $14 \mathrm{TeV}$. In the second part of this work, based on the recent upper limits which have been set on the four top quark cross section by the ATLAS and CMS collaborations from $13 \mathrm{TeV}$ data, we constrain the top quark strong and weak dipole moments. The bounds on the top quark dipole moments are presented using the future LHC prospects for four top quark cross section measurement.
\end{abstract}

DOI: 10.1103/PhysRevD.98.095001

\section{INTRODUCTION}

In proton-proton collisions at the LHC, mostly top quarks are produced in pair via strong interaction [1,2] or singly via weak interactions $[1,2]$. However, the large center-of-mass energy of proton-proton collisions at the LHC opens the possibility to have three $[3,4]$ or four top quark productions. Searches for four top quark production using the Run I data set at $\sqrt{s}=8 \mathrm{TeV}$ have been performed by both the CMS [5,6] and ATLAS $[7,8]$ experiments, with no observed excess of data above the background expectation. The searches of both experiments have been updated using the $13 \mathrm{TeV}$ data using different final states [9-15]. The cross section of the four top quark production at the LHC with the center-of-mass energy of $13 \mathrm{TeV}$ is around $\sigma(p p \rightarrow t \bar{t} t \bar{t})=9 \mathrm{fb}[16,17]$. Within the $\mathrm{SM}$ at leading-order, the three top quarks are produced in association with either a $W$ boson or a jet with a total rate of around $2 \mathrm{fb}[3,4]$. Although three and four top rates are

\footnotetext{
*M_Malekhosseini@semnan.ac.ir

†ghominejad@semnan.ac.ir

Hamzeh.Khanpour@mail.ipm.ir

${ }^{\S}$ Mojtaba@cern.ch
}

Published by the American Physical Society under the terms of the Creative Commons Attribution 4.0 International license. Further distribution of this work must maintain attribution to the author(s) and the published article's title, journal citation, and DOI. Funded by SCOAP ${ }^{3}$. extremely small with respect to the $t \bar{t}[18,19]$ or single top quark production by around five order of magnitudes, these processes are particularly sensitive to new physics (NP) beyond the standard model (SM). Beyond the SM scenarios predict enhancements in $t t \bar{t} X$ and $t \bar{t} t \bar{t}$ production cross section [3,4,20-26]. Vectorlike quarks, supersymmetry (SUSY) with R-parity violation are examples of the BSM scenarios which affect their rates and some have been experimentally studied [5,7,9,11,27-29].

So far, the top quark is the heaviest discovered particle with its mass close to the scale of electroweak symmetry breaking and Yukawa coupling near one, $y_{t} \sim 1$. Therefore, one may expect that possible NP effects would show up in top quark production or decays [30-41]. NP can be seen either directly in new particles production or through the indirect effects via the higher order corrections. Indeed, the observation of indirect indications is important because it provides hints to search for new physics before direct observation. Within the SM framework, the branching fractions of the rare decays of the top quark $t \rightarrow q X$, with $X=g, \gamma, Z$, Higgs and $q=u, c$, are extremely small and are of the order of $10^{-14}-10^{-12}$ [42]. Due to smallness of these branching fractions, the current and future experiments would not be able to measure them. Such transitions in the SM are only possible at loop-level and are significantly suppressed because of the Glashow-Iliopoulos-Maiani (GIM) mechanism [43]. On the other part, it has been found that many SM extensions potentially can relax the suppression in top quark 
FCNC decays from GIM mechanism leading to considerable enhancements for $\mathcal{B}(t \rightarrow q X)$ [42]. This happens because of the appearance of several loop diagrams with new particles mediated inside them. Beyond the SM scenarios like technicolor, SUSY models, two-Higgs doublet models predict much higher branching fractions of the order of $\sim 10^{-10}$ to $10^{-6}$ which are larger than the SM values [42, 44-51]. Searches for FCNC in the top quark sector have been followed by various experiments in the past years, including ALEPH, DELPHI, L3 and OPAL experiments at LEP [52-55], H1 and ZEUS experiments at HERA [56-60], CDF and D0 experiments at the Tevatron [61-65], and CMS and ATLAS experiments at the LHC. The most stringent limits on the top FCNC branching fractions come from the LHC experiments. The CMS collaboration presented the result of their search for the FCNC through single-top-quark production in association with a photon [66]. Based on an integrated luminosity of $19.8 \mathrm{fb}^{-1}$ in $p p$ collisions at a center-of-mass energy of $8 \mathrm{TeV}$, upper limits on $\mathcal{B}(t \rightarrow q \gamma)$ and $\mathcal{B}(t \rightarrow q Z)$ through FCNC single-top-quark production in association with a photon or a $Z$ boson are obtained $[66,67]$. The upper limits on the branching fractions at 95\% CL are

$$
\begin{array}{ll}
\mathcal{B}(t \rightarrow u \gamma)<1.3 \times 10^{-4}, & \mathcal{B}(t \rightarrow c \gamma)<1.70 \times 10^{-3}, \\
\mathcal{B}(t \rightarrow u Z)<2.2 \times 10^{-4}, & \mathcal{B}(t \rightarrow c Z)<4.9 \times 10^{-4} .
\end{array}
$$

The most recent search for the $t q H \mathrm{FCNC}$ has been done by looking at the events with either single top quark FCNC production associated with a Higgs boson and $t \bar{t}$ production with FCNC decay of one of the top quarks [68-70]. The search is based on the Higgs boson decay into a $b \bar{b}$ pair and uses the data corresponding to an integrated luminosity of $35.9 \mathrm{fb}^{-1}$ at $\sqrt{s}=13 \mathrm{TeV}$. The observed upper limits at 95\% CL on the branching fractions of FCNC top quark decays are [69]:

$$
\mathcal{B}(t \rightarrow u H)<4.7 \times 10^{-3}, \quad \mathcal{B}(t \rightarrow c H)<4.7 \times 10^{-3} .
$$

These upper limits are the most stringent ones to date. Among all the FCNC top quark decays, the upper limits on $\mathcal{B}(t \rightarrow q g)$ has been tightly bounded. Using the data collected at the center-of-mass energy of $8 \mathrm{TeV}$ corresponding to an integrated luminosity of $20.3 \mathrm{fb}^{-1}$ by the ATLAS detector, the observed upper limits on the branching fractions are found to be [71]:

$$
\mathcal{B}(t \rightarrow u g)<4.0 \times 10^{-5}, \quad \mathcal{B}(t \rightarrow c g)<2.0 \times 10^{-5} .
$$

The CMS experiment search for the FCNC tqg provides the following limits at the 95\% CL [72]:
$\mathcal{B}(t \rightarrow u g)<2.0 \times 10^{-5}, \quad \mathcal{B}(t \rightarrow c g)<4.1 \times 10^{-4}$.

As it can be seen, the ATLAS experiment has obtained stronger bound on $\mathcal{B}(t \rightarrow c g)$ than the CMS experiment and vice versa for the $\mathcal{B}(t \rightarrow u g)$. So far, there have been a lot of studies on the top quark FCNC in proton-proton, electronpositron and electron-proton colliders [73-84]. In the first part of this work, we perform a search for the FCNC interactions separately at the $t u X$ and $t c X$ vertices at the LHC by looking for events with only three top quark in the final state. We focus on the decay channels in which two same-sign top quarks decay into a $\mathrm{W}$ boson and a $b$-quark, followed by the $\mathrm{W}$ boson decay to an electron or muon and a neutrino. The same-sign dilepton decay channels are considered because it has a clean signature and does not suffer from large background contribution.

In the second part of the work, we study the strong and electroweak dipole moments of the top quark. In the SM, at leading order dipole interactions do not exist, however, electroweak radiative corrections generate both strong and weak dipole moments for the top quark. The size of top quark dipole moments are so small that the LHC would not be able to observe them. But, there are several wellmotivated beyond the SM theories which contribute to these dipoles and lead to sizable enhancements which make the dipoles accessible by the LHC detectors [85-87]. As a consequence, any observation of considerable deviations from zero would be an indication to beyond the SM physics. Here, we investigate the sensitivity of the four top quark production to the weak and strong top quark dipole moments and upper limits are set on them using the present and prospects upper limits on the four top quark production cross section.

The present article is organized as follows: In Sec. II, we present the theoretical framework for describing the top quark FCNC couplings and dipole moments which is based on the effective field theory approach. The sensitivity estimations of three top quark production on FCNC couplings are given in Sec. III. Section IV is dedicated to probe the strong and weak dipole moments using the four top quark production. Finally, Sec. V is dedicated to summarize the results and conclusions.

\section{THEORETICAL FRAMEWORK AND ASSUMPTIONS}

In this paper, we assume that new physics effects in three top quark and four top quark productions are not going to be directly discovered at the LHC (like new heavy degrees of freedom). These kinds of effects could be described by an effective Lagrangian with the following form $[88,89]$ :

$$
\mathcal{L}_{\text {eff }}=\mathcal{L}_{\mathrm{SM}}+\frac{1}{\Lambda^{2}} \sum_{i} c_{i} O_{i}^{d=6}+\text { H.c. },
$$


where $c_{i}$ coefficients are dimensionless by which the new physics effects are parametrized with dimension-six operators $O_{i}$. The scale of new physics is denoted by $\Lambda$ and the $O_{i}$ are a complete set of dimension-six operators which satisfy the SM symmetries, i.e., the Lorentz and the $S U(3) \times S U(2) \times U(1)$ gauge symmetries. Due to the observed excellent agreement between the predications of the SM and data, the deviations from the SM are expected to be small.

There are different dimension-six operators which contribute to the three top and four top quark productions at the LHC. For instance, the impacts of full set of four-fermion qqtt dimension-six operators on the four top production have been studied in Ref. [90]. In this work, the aim is to probe the FCNC interactions (tqg, tqZ, tqq, tqH) coming from dimension-six operators via three top quark production and the effects of dimension-six operators on $g t \bar{t}$ and $Z t \bar{t}$ couplings through the four top quark production at the LHC.

Following on the notation presented in Ref. [88], the operators, $O_{u G \phi}^{23(32)}, O_{u W}^{23(32)}, O_{u B \phi}^{23(32)}, O_{u \phi}^{23(32)}$ contribute to the FCNC couplings and $O_{u G \phi}^{33}, O_{u B \phi}^{33}, O_{u W}^{33}$, and $O_{\phi u}^{33}$ give contributions to $g t \bar{t}$ and $Z t \bar{t}$ interactions. The most general effective Lagrangian describing the FCNC interactions can be parametrized as

$$
\begin{aligned}
\mathcal{L}_{\mathrm{FCNC}}= & \sum_{q=u, c}\left[\frac{g_{s}}{2 m_{t}} \bar{q} \lambda^{a} \sigma^{\mu \nu}\left(\zeta_{q t}^{L} P^{L}+\zeta_{q t}^{R} P^{R}\right) t G_{\mu \nu}^{a}\right. \\
& -\frac{1}{\sqrt{2}} \bar{q}\left(\eta_{q t}^{L} P^{L}+\eta_{q t}^{R} P^{R}\right) t H \\
& -\frac{g_{W}}{2 c_{W}} \bar{q} \gamma^{\mu}\left(X_{q t}^{L} P_{L}+X_{q t}^{R} P_{R}\right) t Z_{\mu} \\
& +\frac{g_{W}}{4 c_{W} m_{Z}} \bar{q} \sigma^{\mu \nu}\left(\kappa_{q t}^{L} P_{L}+\kappa_{q t}^{R} P_{R}\right) t Z_{\mu \nu} \\
& \left.+\frac{e}{2 m_{t}} \bar{q} \sigma^{\mu \nu}\left(\lambda_{q t}^{L} P_{L}+\lambda_{q t}^{R} P_{R}\right) t A_{\mu \nu}\right]+ \text { H.c. },
\end{aligned}
$$

where $\zeta_{q t}, \eta_{q t}, X_{q t}, \kappa_{q t}$, and $\lambda_{q t}$ are the real parameters which determine the strength of FCNC interactions with gluon, Higgs, $Z$, and photon, respectively. At tree-level, in the SM, all the above coefficients are zero, i.e., $\zeta_{q t}^{L}=\zeta_{q t}^{R}=$ $\eta_{q t}^{L}=\eta_{q t}^{R}=X_{q t}^{L}=X_{q t}^{R}=\kappa_{q t}^{L}=\kappa_{q t}^{R}=\lambda_{q t}^{L}=\lambda_{q t}^{R}=0.0$. After the electroweak symmetry breaking, these FCNC parameters are related to the Wilson coefficients of dimension six operators [88]. For example, the FCNC tqg parameters $\zeta_{q t}^{L}$ and $\zeta_{q t}^{R}$ are related to the Wilson coefficients through:

$\zeta_{q t}^{L}=\frac{\sqrt{2} \operatorname{Re}\left(C_{u G \phi}^{32 *}\right) v m_{t}}{g_{s} \Lambda^{2}}, \quad \zeta_{q t}^{R}=\frac{\sqrt{2} \operatorname{Im}\left(C_{u G \phi}^{23}\right) v m_{t}}{g_{s} \Lambda^{2}}$

The effective Lagrangian including dimension-six operators for $g t \bar{t}$ and $Z t \bar{t}$ can be written as:
$\mathcal{L}_{g t \bar{t}}=-g_{s} \overline{\bar{t}} \frac{\lambda^{a}}{2} \gamma^{\mu} t G_{\mu}^{a}-g_{s} \bar{t} \lambda^{a} \frac{i \sigma^{\mu \nu} q_{\nu}}{m_{t}}\left(d_{V}^{g}+i d_{A}^{g} \gamma_{5}\right) t G_{\mu}^{a}$,

and

$$
\begin{aligned}
\mathcal{L}_{Z \bar{t} \bar{t}}= & -\frac{g_{W}}{2 c_{W}} \bar{t} \gamma^{\mu}\left(X_{t t}^{L} P_{L}+X_{t t}^{R} P_{R}-2 s_{W}^{2} Q_{t}\right) t Z_{\mu} \\
& -\frac{g_{W}}{2 c_{W}} \bar{t} \frac{i \sigma^{\mu \nu} q_{\nu}}{m_{Z}}\left(d_{V}^{Z}+i d_{A}^{Z} \gamma_{5}\right) t Z_{\mu} .
\end{aligned}
$$

The couplings $d_{V}^{g(Z)}$ and $d_{A}^{g(Z)}$ are real parameters and are related to strong (weak) magnetic and strong (weak) electric dipole moments of the top quark, respectively. In the SM, at tree-level, $d_{V}^{g(Z)}=0.0$ and $d_{A}^{g(Z)}=0.0$ while the QCD and electroweak corrections at loop level generate very tiny values for the weak and strong dipole moments [91-96]. The values of $X_{t t}^{L}$ and $X_{t t}^{R}$ parameters are equal to one and zero in the SM, respectively.

The most stringent bounds on $d_{V}^{g}$ and $d_{A}^{g}$ are derived from the low energy measurements. In particular, $d_{V}^{g}$ is constrained using the rare $B$-mesons decays [91] and $d_{A}^{g}$ is probed through the neutron electric dipole moment $\left(d_{n}\right)$ [97]. The 95\% CL limits from the low energy experiments are $[91,97]$ :

$$
-3.8 \times 10^{-3} \leq d_{V}^{g} \leq 1.2 \times 10^{-3}, \quad\left|d_{A}^{g}\right| \leq 0.95 \times 10^{-3} .
$$

The combination of the LHC and Tevatron top quark pair cross section measurements leads to the following $95 \% \mathrm{CL}$ regions [41]:

$$
-0.012 \leq d_{V}^{g} \leq 0.023, \quad\left|d_{A}^{g}\right| \leq 0.087 .
$$

As it can be seen, the indirect constraints are tighter than the direct ones by one order of magnitude.

The weak dipole moments $d_{V}^{Z}$ and $d_{A}^{Z}$ have been studied at the LHC and a future electron-positron collider using the $t \bar{t} Z$ production [98]. At the LHC, both $d_{V}^{Z}$ and $d_{A}^{Z}$ are expected to be probed down to the order of 0.15 with $300 \mathrm{fb}^{-1}$ of integrated luminosity of data which would be well-improved to 0.08 using $3000 \mathrm{fb}^{-1}$ integrated luminosity of data. Limits from the electroweak precision data are found to be at the same order. A future $e^{-} e^{+}$collider at the center-of-mass energy of $500 \mathrm{GeV}$ with $500 \mathrm{fb}^{-1}$ would be able to achieve the limits of 0.08 on $\left|d_{A}^{Z}\right|$ and $[-0.02,0.04]$ on the $d_{V}^{Z}[98]$.

\section{SENSITIVITY OF THREE TOP QUARK PRODUCTION TO FCNC COUPLINGS}

In this section, we study the sensitivity of the three top quark production cross section to the FCNC interactions of $t q g, t q \gamma, t q Z$, and $t q H$. In the SM at leading order, similar 
to the single top quark production, three top quark events are produced in association with a light-jet, or a b-jet, or associated with a $W$ boson, i.e.,

$$
\begin{aligned}
& p p \rightarrow t t \bar{t}(t \bar{t} \bar{t})+\text { jet, } \\
& p p \rightarrow t t \bar{t}(t \bar{t} \bar{t})+b \text {-quark, } \\
& p p \rightarrow t t \bar{t}(t \bar{t} \bar{t} \bar{t})+W .
\end{aligned}
$$

The sum of cross section of all processes amounts to around $1.9 \mathrm{fb}$ at the LHC with the center-of-mass energy of $14 \mathrm{TeV}$. The presence of FCNC couplings $t q X, X=g, \gamma, Z, H$, would lead to the production of only three top quark (which does not exist at leading order in SM). Figure 1 shows the lowest-order diagram for the three top quark production from tqg FCNC coupling including the leptonic decays of the $W$ boson from top quarks and hadronic decay of the $W$ boson from antitop quark. The FCNC vertex in this Feynman diagram is denoted by a filled circle. The diagrams for the other FCNC interactions are similar except that the gluon should be replaced by a photon, a Z-boson, or a Higgs boson.

In this work, the search is performed for all FCNC couplings of $t q g, t q \gamma, t q Z$, and $t q H$ independently, i.e., one is switched on at a time. We also do the analysis separately for $q=u$ and $q=c$. We concentrate on a very clean signature with two same-sign leptons, where the lepton could be either an electron or a muon. Therefore, the signal events are generally characterized by the presence of exactly two isolated same-sign charged leptons, large missing transverse energy, and several jets from which three of them come from $b$-quarks. We perform the analysis for 300 and $3000 \mathrm{fb}^{-1}$ of the LHC at the center-of-mass

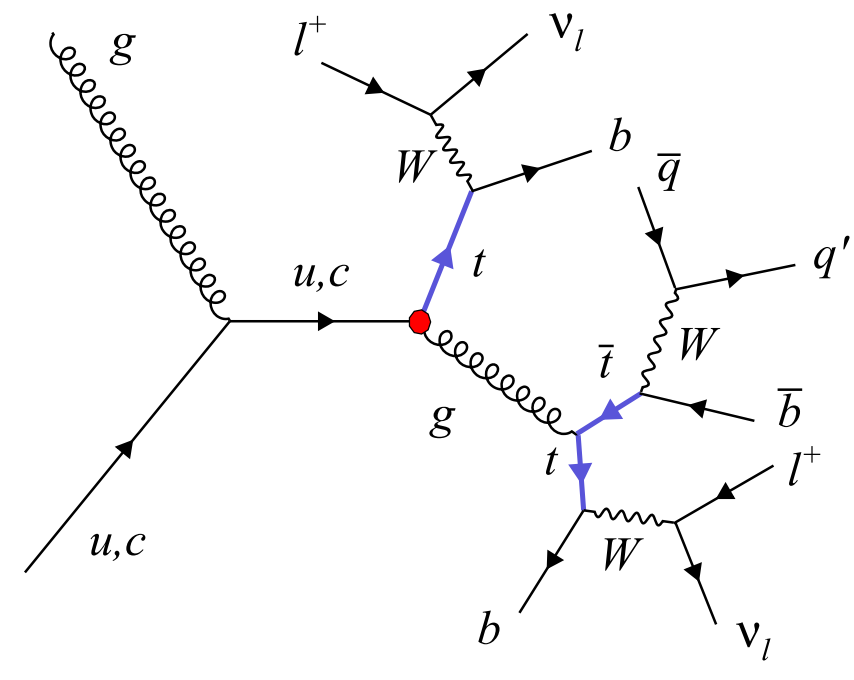

FIG. 1. A representative leading order Feynman diagram of the tqg FCNC contributions to the three top quark production at the LHC including the lepton decay of the $\mathrm{W}$ boson from the top quark decay and hadronic decays of the $\mathrm{W}$ boson from the antitop quark. energy of $14 \mathrm{TeV}$ and present the upper limits on the branching fractions of $\mathcal{B}(t \rightarrow q X)$ at $95 \%$ CL.

\section{A. Event simulation and selection}

In this section, the simulation tools and techniques as well as the event selection and reconstruction are described. The process of signal is taken as three top quarks followed by the leptonic decay of two same-sign top quarks and hadronic decay of the other top quark. As a result, the final state consists of two same-sign charged leptons, at least five jets from which three are originating from $b$-quarks, and missing transverse energy. In this exploratory study, the background processes such as $t \bar{t} Z, t \bar{t} W$, SM four top, $t \bar{t} W W, t \bar{t} Z Z$, and $W W Z$ are considered. For both signal and the background processes, we use MADGRAPH5_AMC@NLO package [99]. It automatically generates the code for obtaining the production rates. The leading order parton distribution functions of NNPDF 3.0 [100] are employed as the input for the calculations and the mass of the top quark is set to $173 \mathrm{GeV}$. To calculate the three top quark cross sections in the presence of the FCNC couplings, the effective Lagrangians introduced above are implemented into the FeynRules package $[101,102]$ and is exported into a UFO module [103] which is connected to MADGRAPH5_AMC@NLO. ${ }^{1}$

To obtain the cross section of three top versus the FCNC couplings, the calculations in the presence of $\zeta_{q t}, \lambda_{q t}, \kappa_{q t}$, and $\eta_{q t}$ are done assuming various values: $\pm 1.0, \pm 2.0$ and fit the resulting cross sections to quadratic polynomials. Considering each FCNC coupling at a time, the cross sections times the related top quark effective coupling strengths are given in the first column of Table I.

As it can be seen, the three top rate has a significant dependence on the tqg FCNC coupling which can be understood by considering the appearance of diagrams like $g u(c) \rightarrow t g \rightarrow t t \bar{t}$. The cross section for the $t q Z$ FCNC coupling are presented for both cases of $\gamma_{\mu}$ and $\sigma_{\mu \nu^{-}}$ couplings. Larger rate is observed for the $\sigma_{\mu \nu}$-coupling which is due to the dependence of the interaction on the $Z$ boson momentum.

To perform the whole simulation chain, PYTHIA [104] is used for showering and hadronization. Jets are reconstructed using the anti- $k_{t}$ algorithm [105] with a cone size of 0.4. DELPHES framework [106] is employed for performing a comprehensive CMS detector [107] response simulation which considers a tracker, calorimeters, and a muon system with a realistic magnetic field configuration of the CMS detector. The $b$-tagging efficiency and misidentification rates for light-flavor quarks are assumed to be dependent on the jet transverse momentum. They are taken as [108]:

\footnotetext{
${ }^{1}$ The UFO file is taken from http://feynrules.irmp.ucl.ac.be/ wiki/GeneralFCNTop.
} 
TABLE I. Cross sections (in fb) of $p p \rightarrow t \bar{t} \bar{t}(\bar{t} \bar{t} t)$ with $\ell=e, \mu$ for five signal scenarios, $t q g, t q H, t q Z\left(\sigma_{\mu \nu}\right)$, $\operatorname{tqZ}\left(\gamma_{\mu}\right)$, and $t q \gamma$ passing sequential cuts explained in text.

\begin{tabular}{lccccc}
\hline \hline$\sqrt{s}=14 \mathrm{TeV}$ & Cross section (in fb) & Cut (I) & Cut (II) & Cut (III) & Cut $(\mathrm{IV})$ \\
\hline$t u g$ & $17614.7\left(\zeta_{t u}\right)^{2}$ & $3621.8\left(\zeta_{t u}\right)^{2}$ & $3418.4\left(\zeta_{t u}\right)^{2}$ & $2650.7\left(\zeta_{t u}\right)^{2}$ & $1794.6\left(\zeta_{t u}\right)^{2}$ \\
$t c g$ & $1239.53\left(\zeta_{t c}\right)^{2}$ & $318.45\left(\zeta_{t c}\right)^{2}$ & $297.95\left(\zeta_{t c}\right)^{2}$ & $229.52\left(\zeta_{t c}\right)^{2}$ & $158.95\left(\zeta_{t c}\right)^{2}$ \\
$t u H$ & $14.49\left(\eta_{t u}\right)^{2}$ & $4.51\left(\eta_{t u}\right)^{2}$ & $4.08\left(\eta_{t u}\right)^{2}$ & $2.96\left(\eta_{t u}\right)^{2}$ & $1.97\left(\eta_{t u}\right)^{2}$ \\
$t c H$ & $1.649\left(\eta_{t c}\right)^{2}$ & $0.55\left(\eta_{t c}\right)^{2}$ & $0.50\left(\eta_{t c}\right)^{2}$ & $0.36\left(\eta_{t c}\right)^{2}$ & $0.24\left(\eta_{t c}\right)^{2}$ \\
$t u Z\left(\gamma_{\mu}\right)$ & $50.56\left(X_{t u}\right)^{2}$ & $14.62\left(X_{t u}\right)^{2}$ & $13.09\left(X_{t u}\right)^{2}$ & $9.48\left(X_{t u}\right)^{2}$ & $6.25\left(X_{t u}\right)^{2}$ \\
$t c Z\left(\gamma_{\mu}\right)$ & $5.505\left(X_{t c}\right)^{2}$ & $1.82\left(X_{t c}\right)^{2}$ & $1.62\left(X_{t c}\right)^{2}$ & $1.17\left(X_{t c}\right)^{2}$ & $0.79\left(X_{t c}\right)^{2}$ \\
$t u Z\left(\sigma_{\mu \nu}\right)$ & $81.49\left(\kappa_{t u}\right)^{2}$ & $22.13\left(\kappa_{t u}\right)^{2}$ & $20.41\left(\kappa_{t u}\right)^{2}$ & $15.32\left(\kappa_{t u}\right)^{2}$ & $10.31\left(\kappa_{t u}\right)^{2}$ \\
$t c Z\left(\sigma_{\mu \nu}\right)$ & $7.154\left(\kappa_{t c}\right)^{2}$ & $2.25\left(\kappa_{t c}\right)^{2}$ & $2.06\left(\kappa_{t c}\right)^{2}$ & $1.53\left(\kappa_{t c}\right)^{2}$ & $1.05\left(\kappa_{t c}\right)^{2}$ \\
$t u \gamma$ & $11.92\left(\lambda_{t u}\right)^{2}$ & $3.22\left(\lambda_{t u}\right)^{2}$ & $2.98\left(\lambda_{t u}\right)^{2}$ & $2.23\left(\lambda_{t u}\right)^{2}$ & $1.50\left(\lambda_{t u}\right)^{2}$ \\
$t c \gamma$ & $1.055\left(\lambda_{t c}\right)^{2}$ & $0.328\left(\lambda_{t c}\right)^{2}$ & $0.301\left(\lambda_{t c}\right)^{2}$ & $0.223\left(\lambda_{t c}\right)^{2}$ & $0.153\left(\lambda_{t c}\right)^{2}$ \\
\hline \hline
\end{tabular}

$b$-tagging efficiency $\epsilon\left[p_{T}\right]$

$$
=0.85 \tanh \left(0.0025 p_{T}\right)\left(\frac{25}{1+0.063 p_{T}}\right),
$$

misidentification rate for $c$-jets $\left[p_{T}\right]$

$$
=0.25 \tanh \left(0.018 p_{T}\right)\left(\frac{1}{1+0.0013 p_{T}}\right),
$$

misidentification rate for light-jets $\left[p_{T}\right]$

$$
=0.01+0.000038 p_{T} \text {. }
$$

The efficiency of $b$-tagging for a jet with $p_{T}=40 \mathrm{GeV}$ is $60 \%$ and the misidentification rates for $c$ - and light-flavor jets are $14 \%$ and $1 \%$, respectively. The events are selected by applying the following simple criteria: (I) Two same-sign charged lepton with $p_{T}>10 \mathrm{GeV},|\eta|<2.5, m_{\ell \ell}>$ $10 \mathrm{GeV}$, (II) Missing Transverse Energy (MET) $>30 \mathrm{GeV}$, (III) At least five-jets with $p_{T}>20 \mathrm{GeV}$, $\left|\eta_{j}\right|<2.5, \Delta R(\ell, j)>0.4, \Delta R\left(j_{1}, j_{2}\right)>0.4$, (IV) At least three $b$-jets. The $b$-jet multiplicity for the $t q g$ and $t q H$ FCNC couplings are depicted in the left panel of Fig. 2. As it is expected, the FCNC signals peak at three while for the backgrounds the peak is at two. The minimum cut of $10 \mathrm{GeV}$

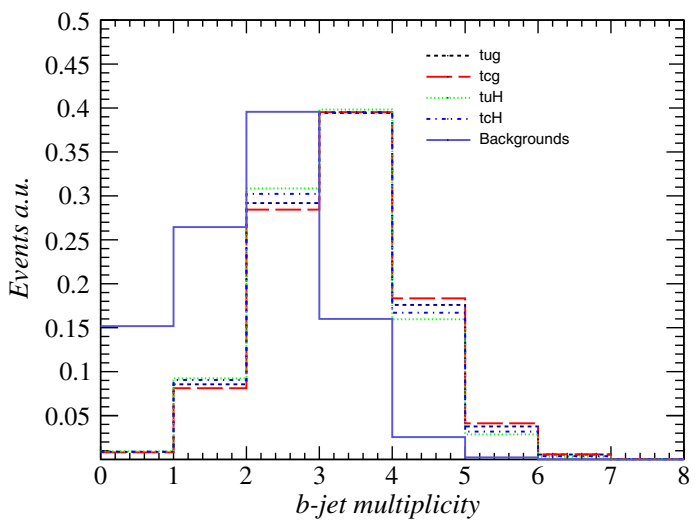

on the invariant mass of the same-sign dilepton is useful to reject events with pairs of same-sign energetic leptons from the heavy hadrons decays. The invariant mass distribution of dilepton is presented in the right side of Fig. 2. There is a shift in the tqg signal scenarios with respect to the background and the $t q H$ signals. This is due to the fact that the $t q g \mathrm{FCNC}$ interactions are momentum dependent. More kinematic cuts and complicated variables could be used to suppress the background contributions and enhance signal significance, however including such variables is beyond the scope of this exploratory analysis and is left to a future work. At this point, it is important to mention that the trigger of such events could be either based on only the presence of same-sign dilepton with loose isolation requirements or based on the existence of same-sign dilepton with lowered transverse momentum thresholds without any isolation requirement, but requiring hadronic activities in the event [109]. The cross sections of various scenarios of FCNC signals and the main background processes after imposing different cuts are presented in Tables I and II, respectively. The cross section values in both tables are given in fb unit. The SM four top, $t \bar{t} W W$, $t \bar{t} Z Z$, and $t \bar{t} H$ are found to have small contributions in the background composition. The sum of the cross section of all these processes after all cuts is found to be $0.02 \mathrm{fb}$. The three top

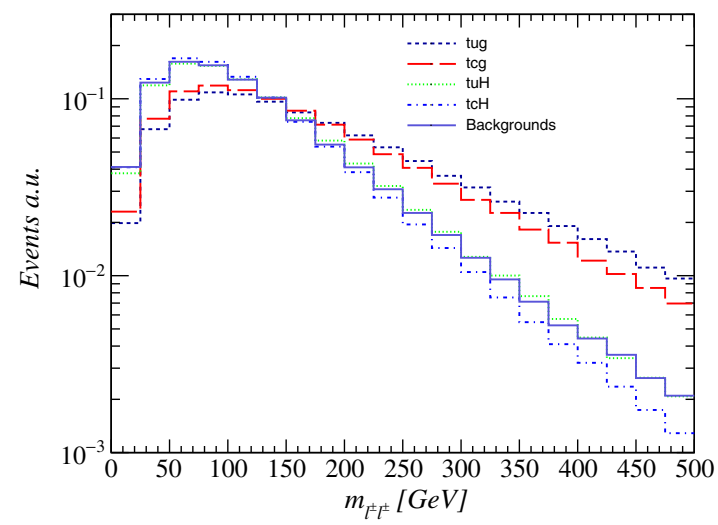

FIG. 2. The $b$-jet multiplicity (left) and invariant mass of dilepton (right) for $t q g$ and $t q H$ signal scenarios obtained from MADGRAPH simulation at leading-order at $\sqrt{s}=14 \mathrm{TeV}$. The sum of all background processes is presented as well. 
TABLE II. Cross section of the main background processes in fb after different set of cuts.

\begin{tabular}{lcc}
\hline \hline Cut & $t \bar{t} Z[\mathrm{fb}]$ & $t \bar{t} W[\mathrm{fb}]$ \\
\hline (I) $2 \ell^{ \pm \pm},\left|\eta_{\ell}\right|<2.5, p_{T}>10 \mathrm{GeV}, m_{\ell \ell}>10 \mathrm{GeV}$ & 1.06 & 2.66 \\
(II) $\mathrm{MET}>30 \mathrm{GeV}$ & 0.88 & 2.38 \\
(III) $n_{j} \geq 5,\left|\eta_{j}\right|<2.5, p_{T}>20, \Delta R(\ell, j)>0.4, \Delta R\left(j_{1}, j_{2}\right)>0.4$ & 0.48 & 0.68 \\
(IV) Number of $b$-jet $\geq 3$ & 0.16 & 0.21 \\
\hline \hline
\end{tabular}

production in association with a jet or a $W$ boson in the SM also could contribute to the background. After all cuts, the rate of SM three top and $W W Z$ are found to be of the order of $10^{-3} \mathrm{fb}$ and $10^{-4} \mathrm{fb}$ which are neglected in this analysis.

At this stage, we go on to set upper limits on the signal rates at $95 \% \mathrm{CL}$. In order to determine the expected limits on the FCNC branching fractions, a Bayesian approach with a flat and positive prior on the cross sections of signals are used. The 95\% CL upper limits on the FCNC signal cross sections are presented in Table III for the integrated luminosity of $300 \mathrm{fb}^{-1}$. The upper limits on signal rates are translated into the upper bounds on the FCNC branching fractions $\mathcal{B}(t \rightarrow q X)$ using the following relations:

$$
\begin{aligned}
\sigma(t u g)(\mathrm{fb}) & =1869.92 \mathcal{B}(t \rightarrow u g), \\
\sigma(t c g)(\mathrm{fb}) & =131.58 \mathcal{B}(t \rightarrow c g) \\
\sigma(t u H)(\mathrm{fb}) & =528.83 \mathcal{B}(t \rightarrow u H), \\
\sigma(t c H)(\mathrm{fb}) & =60.18 \mathcal{B}(t \rightarrow c H) \\
\sigma(t u Z)\left(\gamma_{\mu}\right)(\mathrm{fb}) & =107.57 \mathcal{B}(t \rightarrow u Z), \\
\sigma(t c Z)\left(\gamma_{\mu}\right)(\mathrm{fb}) & =11.71 \mathcal{B}(t \rightarrow c Z)-\gamma_{\mu} \\
\sigma(t u Z)\left(\sigma_{\mu \nu}\right)(\mathrm{fb}) & =220.24 \mathcal{B}(t \rightarrow u Z), \\
\sigma(t c Z)\left(\sigma_{\mu \nu}\right)(\mathrm{fb}) & =19.33 \mathcal{B}(t \rightarrow c Z)-\sigma_{\mu \nu} \\
\sigma(t u \gamma)(\mathrm{fb}) & =27.72 \mathcal{B}(t \rightarrow t u \gamma), \\
\sigma(t c \gamma)(\mathrm{fb}) & =2.45 \mathcal{B}(t \rightarrow t c \gamma) .
\end{aligned}
$$

TABLE III. The expected 95\% CL upper limits on the three top FCNC cross sections for five signal scenarios, tqg, tqH, $\operatorname{tqZ}\left(\sigma_{\mu \nu}\right), t q Z\left(\gamma_{\mu}\right)$, and $t q \gamma$ for the integrated luminosity of $300 \mathrm{fb}^{-1}$.

\begin{tabular}{lc}
\hline \hline Signal & Upper limit on $\sigma\left(\right.$ in fb) for $300 \mathrm{fb}^{-1}$ \\
\hline$\sigma_{95 \%}(t u g)$ & 0.748 \\
$\sigma_{95 \%}(t c g)$ & 0.594 \\
$\sigma_{95 \%}(t u H)$ & 0.550 \\
$\sigma_{95 \%}(t c H)$ & 0.513 \\
$\sigma_{95 \%}(t u Z)\left(\gamma_{\mu}\right)$ & 0.616 \\
$\sigma_{95 \%}(t c Z)\left(\gamma_{\mu}\right)$ & 0.530 \\
$\sigma_{95 \%}(t u Z)\left(\sigma_{\mu \nu}\right)$ & 0.602 \\
$\sigma_{95 \%}(t c Z)\left(\sigma_{\mu \nu}\right)$ & 0.517 \\
$\sigma_{95 \%}(t u \gamma)$ & 0.605 \\
$\sigma_{95 \%}(t c \gamma)$ & 0.525 \\
\hline \hline
\end{tabular}

These formulas are obtained using the functionality of the branching fractions in terms of $\zeta_{q t}, \eta_{q t}, X_{q t}, \kappa_{q t}$, and $\lambda_{q t}$ which could be found in Ref. [110].

The 95\% CL constraints on various FCNC branching fractions are summarized in Table IV for two scenarios of integrated luminosities 300 and $3000 \mathrm{fb}^{-1}$ of data. The results from a recent ATLAS experiment analysis which is based on $t \bar{t}$ process with one of the top quark decays via FCNC and another one decays in standard way are also presented for comparison [111]. As the comparison with the ATLAS limits shows, the three top process would be able to reach similar sensitivity to ATLAS in the tqH FCNC coupling. However, it should be noted that the limits from the three top process could be considerably improved taking into account the other three top quark signatures. In addition, employing more powerful variables and tools (like a multivariate technique [112]) to discriminate signal events from backgrounds would lead to better sensitivities. Taking into account the next to leading order QCD corrections to the signal processes which includes the three top plus a light jet, would significantly tighten the upper bounds on the FCNC branching fractions.

One might worry for the validity of the effective field theory approach used in this analysis. This issue has been studies in several papers such as [114-116]. By assuming the Wilson coefficient to be equal to at most $4 \pi$, one could translate the bounds on the FCNC branching fractions or equivalently the FCNC parameters into a lower limit on the new physics scale, i.e., $\Lambda$. For example, the upper limit on $\mathcal{B}(t \rightarrow u g)$ or correspondingly on $\zeta_{u t}^{L, R}$ leads to a lower bound of $13.4 \mathrm{TeV}$ on $\Lambda$. Such a limit assures that the effective Lagrangian in Eq. (7) is valid with respect to the scale of probed momentum transfers.

\section{SENSITIVITY OF FOUR TOP QUARK PRODUCTION TO THE TOP QUARK WEAK AND STRONG DIPOLE MOMENTS}

In this section, we explore the sensitivity of four top quark production in $p p$ collisions at the center-of-mass energy of $13 \mathrm{TeV}$ to the strong $\left(d_{A, V}^{g}\right)$ and weak $\left(d_{A, V}^{Z}\right)$ top quark electric and magnetic dipole moments. The representative leading order Feynman diagrams including the contributions of strong dipole moments as filled circles are displayed in Fig. 3. 
TABLE IV. The upper limits on the $t q X$ FCNC at $95 \%$ CL obtained at the $\sqrt{s}=14 \mathrm{TeV}$ based on the integrated luminosities of 300 and $3000 \mathrm{fb}^{-1}$. The HL-LHC results from a recent ATLAS experiment study which uses $t \bar{t}$ process are presented for comparison [111].

\begin{tabular}{|c|c|c|c|}
\hline Branching fraction & three top, $300 \mathrm{fb}^{-1}$ & three top, $3 \mathrm{ab}^{-1}$ & other channels, HL-LHC, $3 \mathrm{ab}^{-1}$ \\
\hline $\mathcal{B}(t \rightarrow u H)$ & $1.03 \times 10^{-3}$ & $3.09 \times 10^{-4}$ & $2.4 \times 10^{-4}[111]$ \\
\hline $\mathcal{B}(t \rightarrow c H)$ & $8.52 \times 10^{-3}$ & $2.54 \times 10^{-3}$ & $2.0 \times 10^{-4}[111]$ \\
\hline $\mathcal{B}(t \rightarrow u g)$ & $4.00 \times 10^{-4}$ & $1.19 \times 10^{-4}$ & $\ldots$ \\
\hline $\mathcal{B}(t \rightarrow c g)$ & $4.51 \times 10^{-3}$ & $1.35 \times 10^{-3}$ & . \\
\hline $\mathcal{B}(t \rightarrow u Z)-\sigma_{\mu \nu}$ & $2.73 \times 10^{-3}$ & $8.18 \times 10^{-4}$ & $4.3 \times 10^{-5}[111]$ \\
\hline $\mathcal{B}(t \rightarrow c Z)-\sigma_{\mu \nu}$ & $2.67 \times 10^{-2}$ & $7.98 \times 10^{-3}$ & $5.8 \times 10^{-5}[111]$ \\
\hline $\mathcal{B}(t \rightarrow u Z)-\gamma_{\mu}$ & $5.73 \times 10^{-3}$ & $1.71 \times 10^{-3}$ & $4.3 \times 10^{-5}[111]$ \\
\hline $\mathcal{B}(t \rightarrow c Z)-\gamma_{\mu}$ & $4.52 \times 10^{-2}$ & $1.35 \times 10^{-2}$ & $5.6 \times 10^{-5}[111]$ \\
\hline $\mathcal{B}(t \rightarrow u \gamma)$ & $2.18 \times 10^{-2}$ & $6.53 \times 10^{-3}$ & $2.7 \times 10^{-5}[113]$ \\
\hline $\mathcal{B}(t \rightarrow c \gamma)$ & $2.14 \times 10^{-1}$ & $6.40 \times 10^{-2}$ & $2.0 \times 10^{-4}[113]$ \\
\hline
\end{tabular}

The contributions of the top quark (weak) chromoelectric $\left(d_{A}^{g, Z}\right)$, (weak) chromomagnetic $\left(d_{V}^{g, Z}\right)$ dipole moments, coming from $O_{u W}^{33}$ and $O_{u G \phi}^{33}$ and $O_{u B \phi}^{33}$ operators, to the $t t \bar{t} \bar{t}$ production rate is determined with the MADGRAPH5_AMC@NLO [99]. By considering at most an effective vertex in each diagram, which means up to $O\left(\Lambda^{-2}\right)$, the total four top cross section becomes at most a quadratic function of dipole moments:

$$
\begin{aligned}
& \sigma(p p \rightarrow t \bar{t} t \bar{t})(f b)=\sigma_{\mathrm{SM}}+154.827 \times d_{V}^{g}+3404.44 \times\left(d_{V}^{g}\right)^{2}, \\
& \sigma(p p \rightarrow t \bar{t} t \bar{t})(f b)=\sigma_{\mathrm{SM}}+2731.27 \times\left(d_{A}^{g}\right)^{2}, \\
& \sigma(p p \rightarrow t \bar{t} t \bar{t})(f b)=\sigma_{\mathrm{SM}}-0.689188 \times d_{V}^{Z}+37.0581 \times\left(d_{V}^{Z}\right)^{2}, \\
& \sigma(p p \rightarrow t \bar{t} t \bar{t})(f b)=\sigma_{\mathrm{SM}}+27.962 \times\left(d_{A}^{Z}\right)^{2},
\end{aligned}
$$

where SM four top cross section is denoted by $\sigma_{\mathrm{SM}}$ and the linear terms are the interference between the SM and new

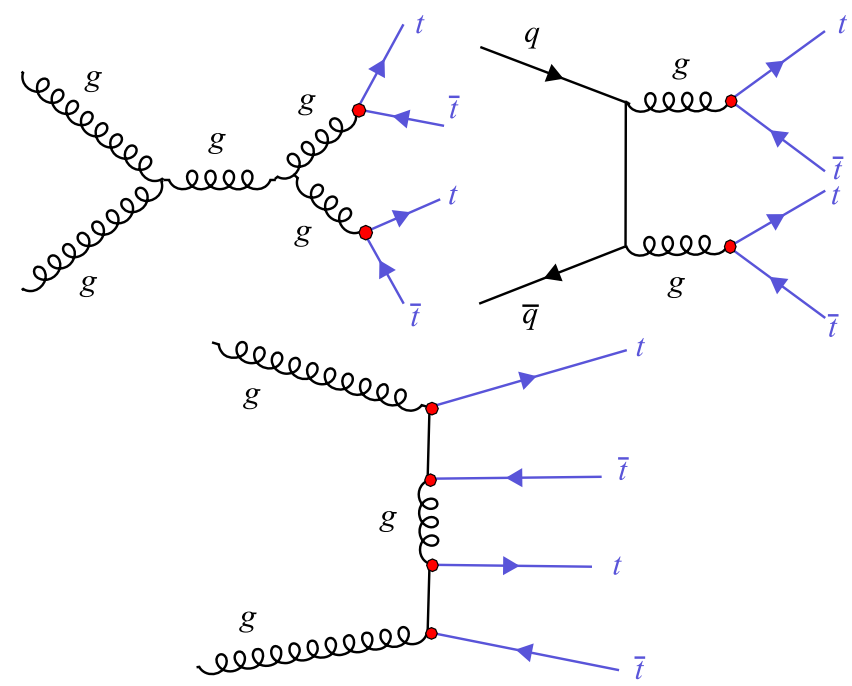

FIG. 3. Illustrative leading order Feynman diagrams for $t \bar{t} t \bar{t}$ production representing the effect of strong dipole moments as filled circles. physics and its contribution is at $\Lambda^{-2}$ order. The quadratic terms in the cross section are corresponding to the power of $\Lambda^{-4}$ which are the first contributing terms for the strong and weak dipole moments. The four top cross section is symmetric with respect to $d_{A}^{g, Z}=0$ because the cross section is a $C P$-even observable. To find the coefficients of cross sections in Eq. (16), the calculations with different values of $d_{A}^{g, Z}$ and $d_{V}^{g, Z}$ are done and fit the obtained cross sections to quadratic polynomials.

In Ref. [12], the CMS experiment has presented the results of a search for four top quark production based on a data set corresponding to an integrated luminosity of $35.9 \mathrm{fb}^{-1}$ in proton-proton collisions at $\sqrt{s}=13 \mathrm{TeV}$. The analysis relies on selecting events containing either a same-sign lepton pair or at least three leptons (e, $\mu$ ) topologies. The observed signal significance is found to be 1.0 standard deviation and the cross section is measured to be $16.9_{-11.4}^{+13.8} \mathrm{fb}$ which is in agreement with the standard model prediction. In Ref. [12], the results are interpreted to limit the top quark Yukawa coupling $\left(y_{t}\right)$ which leads to $y_{t} / y_{t}^{\mathrm{SM}}<2.1$ at the $95 \%$ confidence level.

The ATLAS experiment search for the four top quark production is based on the single electron or muon with large transverse momentum and a high jet multiplicity topology [9]. The analysis has been performed using $3.2 \mathrm{fb}^{-1}$ of pp collisions at the center-of-mass energy of $13 \mathrm{TeV}$ and to improve the search sensitivity, events are classified based on the jet and b-jet multiplicities. An upper limit of $190 \mathrm{fb}$ on the four top cross section (21 times the SM value) is set at the 95\% CL. In the ATLAS study, upper bounds on the four-fermion contact interaction and a universal extra dimensions (UED) model parameters have been set. In our study, we only use the four top cross section measurement done by the CMS experiment that is used as it is the most restrictive results to date.

The upper limits at $95 \% \mathrm{CL}$ on the strong $\left(d_{A, V}^{g}\right)$ and weak $\left(d_{A, V}^{Z}\right)$ dipole moments using the CMS experiment measurement [12], which is the most recent one, are 
TABLE V. Limits on $d_{V}^{g, Z}$ and $d_{A}^{g, Z}$ at 95\% CL corresponding to current and future four top cross section measurements.

\begin{tabular}{lcc}
\hline \hline Coupling & $\begin{array}{c}\text { Current four top } \\
\text { with } 35.6 \mathrm{fb}^{-1}\end{array}$ & $\begin{array}{c}\text { Future four top } \\
\text { with } 300 \mathrm{fb}^{-1}\end{array}$ \\
\hline$d_{V}^{g}$ & {$[-0.20,0.11]$} & {$[-0.07,0.03]$} \\
$d_{A}^{g}$ & {$[-0.16,0.16]$} & {$[-0.05,0.05]$} \\
$d_{V}^{Z}$ & {$[-1.42,1.45]$} & {$[-0.45,0.47]$} \\
$d_{A}^{Z}$ & {$[-1.65,1.65]$} & {$[-0.53,0.53]$} \\
\hline \hline
\end{tabular}

presented in Table V. The resulted bounds are compatible with the ones obtained from top quark pair cross sections at the Tevatron and the LHC. Although the results are looser, this study complements the capabilities of other channels at the LHC. The four top channel would not be able to compete with the $t \bar{t} Z$ and $t \bar{t} Z Z$ channels to probe the weak dipole moments of the top quark.

\section{A. Future prospect for the top quark dipole moments}

To complete our study towards accessing more sensitivity to new physics effect at the LHC, it is important to have an estimate of the sensitivity of the four top quark production using the Run-II LHC reach. In this section, we derive the limits at 95\% CL on the branching fractions of the FCNC transitions and the top quark strong and weak couplings using the possible future reach of the LHC to measure the four top cross section. In Ref. [14], a novel strategy to search for four top production based on the same-sign dilepton and the trilepton channels is presented which allows to avoid the huge backgrounds in the full-hadronic and monoleptonic decay channels. Signal features such as large jet and b-jet multiplicity and a Z-mass veto in the opposite- and sameflavor dilepton spectrum, are used to suppress the main background and increase the ratio of signal-to-background ratio as well as the signal significance. Using the suggested strategy leads to reach the following upper limit on the four top signal strength using $300 \mathrm{fb}^{-1}$ integrated luminosity of data at $\sqrt{s}=13 \mathrm{TeV}$ [14]:

$$
\mu_{t \bar{t} \bar{t} \bar{t}}=\frac{\sigma_{t \bar{t} \bar{t} \bar{t}}^{\exp }}{\sigma_{t \bar{t} \bar{t} \bar{t}}^{\mathrm{SM}}}<1.87
$$

where $\sigma_{t t \bar{t} \bar{t}}^{\exp }$ is the expected cross section of the four top production using $300 \mathrm{fb}^{-1}$ of data. Considering this estimation, we redo our analysis to determine the sensitivity of four top production to the top quark dipole moments. In Table V, the projections of the constraints on the strong and weak dipole moments using the four top quark with $300 \mathrm{fb}^{-1}$ are depicted. The bounds can be translated to a lower limit on the expected new physics scale, which is found to satisfy $\Lambda \gtrsim 4.6 \mathrm{TeV}$. This again ensures the validity of the effective Lagrangian of Eq. (9).

We note that while the indirect constraints on $d_{V}^{g}$ and $d_{A}^{g}$, obtained from the rare B-meson decays and from the neutron electric dipole moment (EDM), and the direct searches from $t \bar{t}$ events are stronger, the results of this search are complementary. Comparison of the achieved sensitivity to $d_{V}^{Z}$ and $d_{A}^{Z}$ with those could be obtained from the LHC and ILC presented in Sec. II, shows that the four top rate does not provide competitive bounds with the $t \bar{t} Z$ channel.

\section{SUMMARY AND CONCLUSIONS}

Due to the very small production rate of the three and four top quark in the SM which are few fb at the LHC, the observation of these processes is quite challenging. However, both the three and four top processes give rise to interesting set of final states depending on various top quarks decay modes. The CMS and ATLAS collaborations have measured the upper limits on four top production cross section in same-sign dilepton and trilepton topologies which does not suffer from large background contributions. These measurements could be directly exploited to confine the NP effects.

Uncommon decays of the top quark with extremely small rates via the flavor violating in the vertices of $t q g$, $t q \mathrm{Z}, t q \gamma$, and $t q H$ are attracting much attention as they are considerably sensitive to several SM extensions. The predictions for the branching fractions of the rare decay modes $t \rightarrow q X, X=g, Z, \gamma, H$ and $q=u, c$, in the SM are expected to be quite small so that they are unobservable at the LHC. However, new physics scenarios predict significant enhancement in the rare top quark branching fractions by many order of magnitudes. Consequently, observation of such decay modes would indicate the existence of beyond the SM.

In this work, we demonstrate that the three top quark production is sensitive to the FCNC couplings. By performing a realistic detector simulation and the main background processes in the same-sign dilepton channel, upper limits on the FCNC branching fractions are obtained. Upper limits of the order of $10^{-4}$ at $95 \% \mathrm{CL}$ on the branching fractions of $t \rightarrow q H, t \rightarrow q g$, and $t \rightarrow q Z$ are set. We also examine the sensitivity of the four top cross section to the strong and weak top quark dipole moments. Upper limits are set on the top quark dipole moments which are compatible with the other bounds extracted from the other processes at the LHC.

\section{ACKNOWLEDGMENTS}

M. M. and M. G. thank the University of Semnan for financial support of this project. H. K. is thankful to the University of Science and Technology of Mazandaran, and School of Particles and Accelerators, Institute for Research in Fundamental Sciences (IPM) for financial support provided for this research. M. M. N. would like to appreciate the Iran National Science Foundation (INSF) for the financial support. 
[1] A. M. Sirunyan et al. (CMS Collaboration), Cross section measurement of $t$-channel single top quark production in $p p$ collisions at $\sqrt{s}=13 \mathrm{TeV}$, Phys. Lett. B 772, 752 (2017).

[2] CMS Collaboration, Measurement of the production cross section for single top quarks in association with $W$ bosons in $p p$ collisions at $\sqrt{s}=13 \mathrm{TeV}$, Report No. CMS-PASTOP-17-018.

[3] V. Barger, W. Y. Keung, and B. Yencho, Triple-top signal of new physics at the LHC, Phys. Lett. B 687, 70 (2010).

[4] C. R. Chen, Searching for new physics with triple-top signal at the LHC, Phys. Lett. B 736, 321 (2014).

[5] V. Khachatryan et al. (CMS Collaboration), Search for standard model production of four top quarks in the lepton + jets channel in $p p$ collisions at $\sqrt{s}=8 \mathrm{TeV}$, J. High Energy Phys. 11 (2014) 154.

[6] S. Chatrchyan et al. (CMS Collaboration), Search for new physics in events with same-sign dileptons and jets in $p p$ collisions at $\sqrt{s}=8 \mathrm{TeV}$, J. High Energy Phys. 01 (2014) 163; Erratum, J. High Energy Phys. 01 (2015) 14.

[7] G. Aad et al. (ATLAS Collaboration), Search for production of vector-like quark pairs and of four top quarks in the lepton-plus-jets final state in $p p$ collisions at $\sqrt{s}=8 \mathrm{TeV}$ with the ATLAS detector, J. High Energy Phys. 08 (2015) 105.

[8] G. Aad et al. (ATLAS Collaboration), Analysis of events with $b$-jets and a pair of leptons of the same charge in $p p$ collisions at $\sqrt{s}=8 \mathrm{TeV}$ with the ATLAS detector, J. High Energy Phys. 10 (2015) 150.

[9] ATLAS Collaboration, Search for four top-quark production in final states with one charged lepton and multiple jets using $3.2 \mathrm{fb}^{-1}$ of proton-proton collisions at $\sqrt{\mathrm{s}}=$ $13 \mathrm{TeV}$ with the ATLAS detector at the LHC, Report No. ATLAS-CONF-2016-020.

[10] A. M. Sirunyan et al. (CMS Collaboration), Search for standard model production of four top quarks in protonproton collisions at $\sqrt{s}=13 \mathrm{TeV}$, Phys. Lett. B 772, 336 (2017).

[11] CMS Collaboration, Search for standard model production of four top quarks in proton-proton collisions at $13 \mathrm{TeV}$, Report No. CMS-PAS-TOP-16-016.

[12] A. M. Sirunyan et al. (CMS Collaboration), Search for standard model production of four top quarks with samesign and multilepton final states in proton-proton collisions at $\sqrt{s}=13$ TeV, Eur. Phys. J. C 78, 140 (2018).

[13] L. Beck (CMS Collaboration), Search for the production of four top quarks at the CMS experiment at $\sqrt{s}=13 \mathrm{TeV}$, arXiv:1611.09607.

[14] E. Alvarez, D. A. Faroughy, J. F. Kamenik, R. Morales, and A. Szynkman, Four tops for LHC, Nucl. Phys. B915, 19 (2017).

[15] CMS Collaboration, Search for the standard model production of four top quarks with same-sign and multilepton final states in proton-proton collisions at $\sqrt{s}=13 \mathrm{TeV}$, Report No. CMS-PAS-TOP-17-009.

[16] R. Frederix, D. Pagani, and M. Zaro, Large NLO corrections in $t \bar{t} W^{ \pm}$and $t \bar{t} t \bar{t}$ hadroproduction from supposedly subleading EW contributions, J. High Energy Phys. 02 (2018) 031.
[17] F. Maltoni, D. Pagani, and I. Tsinikos, Associated production of a top-quark pair with vector bosons at NLO in QCD: impact on $t \bar{t} H$ searches at the LHC, J. High Energy Phys. 02 (2016) 113.

[18] M. Aaboud et al. (ATLAS Collaboration), Measurements of top-quark pair differential cross-sections in the lepton + jets channel in $p p$ collisions at $\sqrt{s}=13 \mathrm{TeV}$ using the ATLAS detector, J. High Energy Phys. 11 (2017) 191.

[19] M. Aaboud et al. (ATLAS Collaboration), Measurements of top-quark pair differential cross-sections in the $e \mu$ channel in $p p$ collisions at $\sqrt{s}=13 \mathrm{TeV}$ using the ATLAS detector, Eur. Phys. J. C 77, 292 (2017).

[20] G. Cacciapaglia, R. Chierici, A. Deandrea, L. Panizzi, S. Perries, and S. Tosi, Four tops on the real projective plane at LHC, J. High Energy Phys. 10 (2011) 042.

[21] J. A. Aguilar-Saavedra and J. Santiago, Four tops and the $t \bar{t}$ forward-backward asymmetry, Phys. Rev. D 85, 034021 (2012).

[22] S. Kanemura, H. Yokoya, and Y. J. Zheng, Searches for additional Higgs bosons in multi-top-quarks events at the LHC and the International linear collider, Nucl. Phys. B898, 286 (2015).

[23] A. Deandrea and N. Deutschmann, Multi-tops at the LHC, J. High Energy Phys. 08 (2014) 134.

[24] T. Gregoire, E. Katz, and V. Sanz, Four top quarks in extensions of the standard model, Phys. Rev. D 85, 055024 (2012).

[25] G. Bevilacqua and M. Worek, Constraining BSM Physics at the LHC: Four top final states with NLO accuracy in perturbative QCD, J. High Energy Phys. 07 (2012) 111.

[26] C. Han, N. Liu, L. Wu, and J. M. Yang, Probing topcolorassisted technicolor from top charge asymmetry and tripletop production at the LHC, Phys. Lett. B 714, 295 (2012).

[27] ATLAS Collaboration, Search for new physics using events with $b$-jets and a pair of same charge leptons in $3.2 \mathrm{fb}^{-1}$ of $p p$ collisions at $\sqrt{s}=13 \mathrm{TeV}$ with the ATLAS detector, Report No. ATLAS-CONF-2016-032.

[28] G. Aad et al. (ATLAS Collaboration), Search for supersymmetry at $\sqrt{s}=13 \mathrm{TeV}$ in final states with jets and two same-sign leptons or three leptons with the ATLAS detector, Eur. Phys. J. C 76, 259 (2016).

[29] CMS Collaboration, Search for SUSY with multileptons in $13 \mathrm{TeV}$ data, Report No. CMS-PAS-SUS-16-022.

[30] U. Husemann, Top-quark physics: Status and prospects, Prog. Part. Nucl. Phys. 95, 48 (2017).

[31] D. Barducci, M. Fabbrichesi, and A. Tonero, Constraints on top quark nonstandard interactions from Higgs and $t \bar{t}$ production cross sections, Phys. Rev. D 96, 075022 (2017).

[32] M. Schulze and Y. Soreq, Pinning down electroweak dipole operators of the top quark, Eur. Phys. J. C 76, 466 (2016).

[33] A. Buckley, C. Englert, J. Ferrando, D. J. Miller, L. Moore, M. Russell, and C.D. White, Constraining top quark effective theory in the LHC Run II era, J. High Energy Phys. 04 (2016) 015.

[34] N. Arkani-Hamed, T. Han, M. Mangano, and L. T. Wang, Physics opportunities of a $100 \mathrm{TeV}$ proton-proton collider, Phys. Rep. 652, 1 (2016). 
[35] Y. C. Guo, C. X. Yue, and S. Yang, Search for anomalous couplings via single top quark production in association with a photon at LHC, Eur. Phys. J. C 76, 596 (2016).

[36] B. Lillie, J. Shu, and T. M. P. Tait, Top compositeness at the Tevatron and LHC, J. High Energy Phys. 04 (2008) 087.

[37] G. Cortiana, Top-quark mass measurements: Review and perspectives, Rev. Phys. 1, 60 (2016).

[38] E. Boos, O. Brandt, D. Denisov, S. Denisov, and P. Grannis, The top quark (20 years after its discovery), Usp. Fiz. Nauk 185, 1241 (2015) [Phys. Usp. 58, 1133 (2015)].

[39] M. Cristinziani and M. Mulders, Top-quark physics at the large hadron collider, J. Phys. G 44, 063001 (2017).

[40] A. Escamilla, A. O. Bouzas, and F. Larios, Single top production at linear $e^{-} e^{+}$colliders, Phys. Rev. D 97, 033004 (2018).

[41] J. A. Aguilar-Saavedra, B. Fuks, and M. L. Mangano, Pinning down top dipole moments with ultra-boosted tops, Phys. Rev. D 91, 094021 (2015).

[42] K. Agashe et al. (Top Quark Working Group), Working group report: Top quark, arXiv:1311.2028.

[43] S. L. Glashow, J. Iliopoulos, and L. Maiani, Weak interactions with Lepton-Hadron symmetry, Phys. Rev. D 2, 1285 (1970).

[44] S. Bejar, J. Guasch, D. Lopez-Val, and J. Sola, FCNCinduced heavy-quark events at the LHC from supersymmetry, Phys. Lett. B 668, 364 (2008).

[45] J. Cao, Z. Heng, L. Wu, and J. M. Yang, $R$-parity violating effects in top quark FCNC productions at LHC, Phys. Rev. D 79, 054003 (2009).

[46] U. K. Dey and T. Jha, Rare top decays in minimal and nonminimal universal extra dimension models, Phys. Rev. D 94, 056011 (2016).

[47] A. Arhrib, Top and Higgs flavor changing neutral couplings in two Higgs doublets model, Phys. Rev. D 72, 075016 (2005).

[48] R. Gaitan, J. H. M. de Oca, E. A. Garces, and R. Martinez, Rare top decay $t \rightarrow c \gamma$ with flavor changing neutral scalar interactions in two Higgs doublet model, Phys. Rev. D 94, 094038 (2016).

[49] B. Yang, N. Liu, and J. Han, Top quark flavor-changing neutral-current decay to a $125 \mathrm{GeV}$ Higgs boson in the littlest Higgs model with $T$ parity, Phys. Rev. D 89, 034020 (2014).

[50] J. L. Diaz-Cruz, J. Hernandez-Sanchez, S. Moretti, R. Noriega-Papaqui, and A. Rosado, Yukawa textures and charged Higgs boson phenomenology in the 2HDM-III, Phys. Rev. D 79, 095025 (2009).

[51] J. J. Cao, G. Eilam, M. Frank, K. Hikasa, G. L. Liu, I. Turan, and J. M. Yang, SUSY-induced FCNC top-quark processes at the large hadron collider, Phys. Rev. D 75, 075021 (2007).

[52] G. Abbiendi et al. (OPAL Collaboration), Search for single top quark production at LEP-2, Phys. Lett. B 521, 181 (2001).

[53] P. Achard et al. (L3 Collaboration), Search for single top production at LEP, Phys. Lett. B 549, 290 (2002).

[54] J. Abdallah et al. (DELPHI Collaboration), Search for single top production via FCNC at LEP at $\sqrt{s}=189-\mathrm{GeV}$ to 208-GeV, Phys. Lett. B 590, 21 (2004).
[55] A. Heister et al. (ALEPH Collaboration), Search for single top production in $e^{+} e^{-}$collisions at $\sqrt{s}$ up to $209-\mathrm{GeV}$, Phys. Lett. B 543, 173 (2002).

[56] S. Moretti and K. Odagiri, Single top production at future ep colliders, Phys. Rev. D 57, 3040 (1998).

[57] S. Chekanov et al. (ZEUS Collaboration), Search for single top production in ep collisions at HERA, Phys. Lett. B 559, 153 (2003).

[58] A. Aktas et al. (H1 Collaboration), Search for single top quark production in ep collisions at HERA, Eur. Phys. J. C 33, 9 (2004).

[59] H. Abramowicz et al. (ZEUS Collaboration), Search for single-top production in ep collisions at HERA, Phys. Lett. B 708, 27 (2012).

[60] F. D. Aaron et al. (H1 Collaboration), Search for single top quark production at HERA, Phys. Lett. B 678, 450 (2009).

[61] F. Abe et al. (CDF Collaboration), Search for FlavorChanging Neutral Current Decays of the Top Quark in $p \bar{p}$ Collisions at $\sqrt{s}=1.8 \mathrm{TeV}$, Phys. Rev. Lett. 80, 2525 (1998).

[62] T. Aaltonen et al. (CDF Collaboration), Search for the Flavor Changing Neutral Current Decay $t \rightarrow Z q$ in $p \bar{p}$ Collisions at $\sqrt{s}=1.96 \mathrm{TeV}$, Phys. Rev. Lett. 101, 192002 (2008).

[63] V. M. Abazov et al. (D0 Collaboration), Search for flavor changing neutral currents in decays of top quarks, Phys. Lett. B 701, 313 (2011).

[64] V. M. Abazov et al. (D0 Collaboration), Search for Production of Single Top Quarks via tcg and tug Flavor-Changing Neutral Current Couplings, Phys. Rev. Lett. 99, 191802 (2007).

[65] T. Aaltonen et al. (CDF Collaboration), Search for TopQuark Production via Flavor-Changing Neutral Currents in $W+1$ Jet Events at CDF, Phys. Rev. Lett. 102, 151801 (2009).

[66] V. Khachatryan et al. (CMS Collaboration), Search for anomalous single top quark production in association with a photon in pp collisions at $\sqrt{s}=8 \mathrm{TeV}$, J. High Energy Phys. 04 (2016) 035.

[67] A. M. Sirunyan et al. (CMS Collaboration), Search for associated production of a $Z$ boson with a single top quark and for $t Z$ flavour-changing interactions in $p p$ collisions at $\sqrt{s}=8 \mathrm{TeV}$, J. High Energy Phys. 07 (2017) 003.

[68] M. Aaboud et al. (ATLAS Collaboration), Search for top quark decays $t \rightarrow q H$, with $H \rightarrow \gamma \gamma$, in $\sqrt{s}=13 \mathrm{TeV} p p$ collisions using the ATLAS detector, J. High Energy Phys. 10 (2017) 129.

[69] CMS Collaboration, Search for the flavor-changing interactions of the top quark with the Higgs boson in $H \rightarrow b \bar{b}$ channel at $\sqrt{s}=13 \mathrm{TeV}$, Report No. CMS-PAS-TOP-17003.

[70] G. Aad et al. (ATLAS Collaboration), Search for flavourchanging neutral current top quark decays $t \rightarrow H q$ in $p p$ collisions at $\sqrt{s}=8 \mathrm{TeV}$ with the ATLAS detector, J. High Energy Phys. 12 (2015) 061.

[71] G. Aad et al. (ATLAS Collaboration), Search for single top-quark production via flavour-changing neutral currents at $8 \mathrm{TeV}$ with the ATLAS detector, Eur. Phys. J. C 76, 55 (2016). 
[72] V. Khachatryan et al. (CMS Collaboration), Search for anomalous $W t b$ couplings and flavour-changing neutral currents in $t$-channel single top quark production in $\mathrm{pp}$ collisions at $\sqrt{s}=7$ and $8 \mathrm{TeV}$, J. High Energy Phys. 02 (2017) 028.

[73] S. Khatibi and M. M. Najafabadi, Probing the anomalous FCNC interactions in top-Higgs final state and charge ratio approach, Phys. Rev. D 89, 054011 (2014).

[74] S. Khatibi and M. Mohammadi Najafabadi, Constraints on top quark flavor changing neutral currents using diphoton events at the LHC, Nucl. Phys. B909, 607 (2016).

[75] S. M. Etesami and M. M. Najafabadi, Study of anomalous top quark FCNC interactions via $t W$-channel of single top, Phys. Rev. D 81, 117502 (2010).

[76] G. Durieux, F. Maltoni, and C. Zhang, Global approach to top-quark flavor-changing interactions, Phys. Rev. D 91, 074017 (2015).

[77] I. T. Cakir, A. Yilmaz, H. Denizli, A. Senol, H. Karadeniz, and O. Cakir, Probing the anomalous FCNC couplings at large hadron electron collider, Adv. High Energy Phys. 2017, 1572053 (2017).

[78] H. Denizli, A. Senol, A. Yilmaz, I. T. Cakir, H. Karadeniz, and O. Cakir, Top quark FCNC couplings at future circular hadron electron colliders, Phys. Rev. D 96, 015024 (2017).

[79] A. Senol, Anomalous Higgs couplings at the LHeC, Nucl. Phys. B873, 293 (2013).

[80] O. Cakir, I. T. Cakir, A. Senol, and A. T. Tasci, Search for top quark FCNC couplings in $Z$ ' models at the LHC and CLIC, Eur. Phys. J. C 70, 295 (2010).

[81] H. Khanpour, S. Khatibi, M. K. Yanehsari, and M. M. Najafabadi, Single top quark production as a probe of anomalous $t q \gamma$ and $t q Z$ couplings at the FCC-ee, Phys. Lett. B 775, 25 (2017).

[82] H. Hesari, H. Khanpour, and M. M. Najafabadi, Direct and indirect searches for top-Higgs FCNC couplings, Phys. Rev. D 92, 113012 (2015).

[83] J. A. Aguilar-Saavedra, Ultraboosted $Z t$ and $\gamma t$ production at the HL-LHC and FCC-hh, Eur. Phys. J. C 77, 769 (2017).

[84] J. F. Shen, Y. Q. Li, and Y. B. Liu, Searches for anomalous tqZ couplings from the trilepton signal of $t Z$ associated production at the $14 \mathrm{TeV}$ LHC, Phys. Lett. B 776, 391 (2018).

[85] T. Ibrahim and P. Nath, The top quark electric dipole moment in an MSSM extension with vector like multiplets, Phys. Rev. D 82, 055001 (2010).

[86] T. Ibrahim and P. Nath, The chromoelectric dipole moment of the top quark in models with vector like multiplets, Phys. Rev. D 84, 015003 (2011).

[87] B. Yang, J. Han, and X. Zhang, Production of $Z t \bar{t}$ and $Z t \bar{T}$ in the littlest Higgs model with $T$-parity at high energy colliders, Eur. Phys. J. C 73, 2639 (2013).

[88] J. A. Aguilar-Saavedra, A minimal set of top anomalous couplings, Nucl. Phys. B812, 181 (2009).

[89] J. A. Aguilar-Saavedra, A minimal set of top-Higgs anomalous couplings, Nucl. Phys. B821, 215 (2009).

[90] C. Zhang, Constraining qqtt operators from four top production: A case for enhanced EFT sensitivity, Chin. Phys. C 42, 023104 (2018).
[91] R. Martinez and J. A. Rodriguez, The anomalous chromomagnetic dipole moment of the top quark in the standard model and beyond, Phys. Rev. D 65, 057301 (2002).

[92] R. Martinez, M. A. Perez, and N. Poveda, Chromomagnetic dipole moment of the top quark revisited, Eur. Phys. J. C 53, 221 (2008).

[93] J. Bernabeu, D. Comelli, L. Lavoura, and J. P. Silva, Weak magnetic dipole moments in two Higgs doublet models, Phys. Rev. D 53, 5222 (1996).

[94] Y. T. Chien, V. Cirigliano, W. Dekens, J. de Vries, and E. Mereghetti, Direct and indirect constraints on CP-violating Higgs-quark and Higgs-gluon interactions, J. High Energy Phys. 02 (2016) 011.

[95] W. Hollik, J. I. Illana, S. Rigolin, C. Schappacher, and D. Stockinger, Top dipole form-factors and loop induced $C P$ violation in supersymmetry, Nucl. Phys. B551, 3 (1999); Erratum, Nucl. Phys. B557, 407(E) (1999).

[96] K. Agashe, G. Perez, and A. Soni, Collider signals of top quark flavor violation from a warped extra dimension, Phys. Rev. D 75, 015002 (2007).

[97] J. F. Kamenik, M. Papucci, and A. Weiler, Constraining the dipole moments of the top quark, Phys. Rev. D 85, 071501 (2012); Erratum, Phys. Rev. D 88, 039903(E) (2013).

[98] R. Röntsch and M. Schulze, Probing top- $Z$ dipole moments at the LHC and ILC, J. High Energy Phys. 08 (2015) 044.

[99] J. Alwall, R. Frederix, S. Frixione, V. Hirschi, F. Maltoni, O. Mattelaer, H.-S. Shao, T. Stelzer, P. Torrielli, and M. Zaro, The automated computation of tree-level and nextto-leading order differential cross sections, and their matching to parton shower simulations, J. High Energy Phys. 07 (2014) 079.

[100] R. D. Ball et al. (NNPDF Collaboration), Parton distributions for the LHC Run II, J. High Energy Phys. 04 (2015) 040.

[101] A. Alloul, N. D. Christensen, C. Degrande, C. Duhr, and B. Fuks, FeynRules 2.0-A complete toolbox for tree-level phenomenology, Comput. Phys. Commun. 185, 2250 (2014).

[102] C. Duhr and B. Fuks, A superspace module for the FeynRules package, Comput. Phys. Commun. 182, 2404 (2011).

[103] C. Degrande, C. Duhr, B. Fuks, D. Grellscheid, O. Mattelaer, and T. Reiter, UFO-The universal FeynRules output, Comput. Phys. Commun. 183, 1201 (2012).

[104] T. Sjostrand, L. Lonnblad, S. Mrenna, and P. Z. Skands, Pythia 6.3 physics and manual, arXiv:hep-ph/0308153.

[105] M. Cacciari, G. P. Salam, and G. Soyez, The anti- $k(t)$ jet clustering algorithm, J. High Energy Phys. 04 (2008) 063.

[106] J. de Favereau, C. Delaere, P. Demin, A. Giammanco, V. Lemaître, A. Mertens, and M. Selvaggi (DELPHES 3 Collaboration), A modular framework for fast simulation of a generic collider experiment, J. High Energy Phys. 02 (2014) 057.

[107] S. Chatrchyan et al. (CMS Collaboration), The CMS experiment at the CERN LHC, J. Instrum. 3, S08004 (2008).

[108] S. Chatrchyan et al. (CMS Collaboration), Identification of $b$-quark jets with the CMS experiment, J. Instrum. 8, P04013 (2013). 
[109] V. Khachatryan et al. (CMS Collaboration), Search for new physics in same-sign dilepton events in proton-proton collisions at $\sqrt{s}=13 \mathrm{TeV}$, Eur. Phys. J. C 76, 439 (2016).

[110] J. A. Aguilar-Saavedra, Top flavour-changing neutral interactions: theoretical expectations and experimental detection, Acta Phys. Pol. B 35, 2695 (2004).

[111] ATLAS Collaboration, Expected sensitivity of ATLAS to FCNC top quark decays $t \rightarrow \mathrm{Zu}$ and $t \rightarrow H q$ at the high Luminosity LHC, Report No. ATL-PHYS-PUB-2016-019.

[112] A. Hocker et al., TMVA-Toolkit for multivariate data analysis, arXiv:physics/0703039.

[113] CMS Collaboration, ECFA 2016: Prospects for selected standard model measurements with the CMS experiment at the high-Luminosity LHC, Report No. CMS-PAS-FTR16-006.

[114] C. Englert and M. Spannowsky, Effective theories and measurements at colliders, Phys. Lett. B 740, 8 (2015).

[115] R. Contino, A. Falkowski, F. Goertz, C. Grojean, and F. Riva, On the validity of the effective field theory approach to SM precision tests, J. High Energy Phys. 07 (2016) 144.

[116] M. Farina, G. Panico, D. Pappadopulo, J. T. Ruderman, R. Torre, and A. Wulzer, Energy helps accuracy: Electroweak precision tests at hadron colliders, Phys. Lett. B 772, 210 (2017). 